Proyecciones Journal of Mathematics

Vol. 37, $\mathrm{N}^{\circ}$ 4, pp. 713-730, December 2018.

Universidad Católica del Norte

Antofagasta - Chile

\title{
On rough convergence of triple sequence spaces of Bernstein-Stancu operators of fuzzy numbers defined by a metric function
}

\author{
M. Jeyaram Bharathi \\ Hindustan Institute of Technology and Science, India \\ S. Velmurugan \\ Hindustan Institute of Technology and Science, India \\ A. Esi \\ Adiyaman University, Turkey \\ and \\ N. Subramanian \\ SASTRA University, India \\ Received : February 2018. Accepted : March 2018
}

\begin{abstract}
We define the concept of rough limit set of a triple sequence space of Bernstein-Stancu polynomials of fuzzy numbers and obtain the relation between the set of rough limit and the extreme limit points of a triple sequence space of Bernstein-Stancu polynomials of fuzzy numbers. Finally, we investigate some properties of the rough limit set of Bernstein-Stancu polynomials.
\end{abstract}

Keywords : Triple sequences, rough convergence, closed and convex, cluster points and rough limit points, fuzzy numbers,Bernstein-Stancu polynomials.

2010 Mathematics Subject Classification : 40F05, 40J05, 40G05. 


\section{Introduction}

The idea of rough convergence was first introduced by Phu [12-14] in finite dimensional normed spaces. He showed that the set $L I M_{x}^{r}$ is bounded, closed and convex; and he introduced the notion of rough Cauchy sequence. He also investigated the relations between rough convergence and other convergence types and the dependence of $L I M_{x}^{r}$ on the roughness of degree $r$.

Aytar [1] studied of rough statistical convergence and defined the set of rough statistical limit points of a sequence and obtained two statistical convergence criteria associated with this set and prove that this set is closed and convex. Also, Aytar [2] studied that the $r$ - limit set of the sequence is equal to intersection of these sets and that $r-$ core of the sequence is equal to the union of these sets. Dündar and Cakan [11] investigated of rough ideal convergence and defined the set of rough ideal limit points of a sequence The notion of $I$ - convergence of a triple sequence spaces which is based on the structure of the ideal $I$ of subsets of $\mathbf{N} \times \mathbf{N} \times \mathbf{N}$, where $\mathbf{N}$ is the set of all natural numbers, is a natural generalization of the notion of convergence and statistical convergence.

Let $K$ be a subset of the set of positive integers $\mathbf{N} \times \mathbf{N} \times \mathbf{N}$ and let us denote the set $K_{i k \ell}=\{(m, n, k) \in K: m \leq i, n \leq j, k \leq \ell\}$. Then the natural density of $K$ is given by

$$
\delta(K)=\lim _{i, j, \ell \rightarrow \infty} \frac{\left|K_{i j \ell}\right|}{i j \ell},
$$

where $\left|K_{i j \ell}\right|$ denotes the number of elements in $K_{i j \ell}$.

First applied the concept of $(p, q)$-calculus in approximation theory and introduced the (p, q)-analogue of Bernstein operators. Later, based on (p, q)-integers, some approximation results for Bernstein-Stancu operators, Bernstein- Kantorovich operators, $(p, q)$-Lorentz operators, Bleimann-Butzer and Hahn operators and Bernstein-Shurer operators etc.

Very recently, Khalid et al. have given a nice application in computeraided geometric design and applied these Bernstein basis for construction of $(p, q)$-Bezier curves and surfaces based on $(p, q)$ - integers which is further generalization of $q$-Bezier curves and surfaces. 
Motivated by the above mentioned work on $(p, q)$-approximation and its application, in this paper we study statistical approximation properties of Bernstein-Stancu Operators based on $(p, q)$-integers.

Now we recall some basic definitions about $(p, q)$-integers. For any $u, v, w \in \mathbf{N}^{3}$, the $(p, q)$-integer $[u v w]_{p, q}$ is defined by

$$
[0]_{p, q}:=0 \text { and }[u v w]_{p, q}=\frac{p^{u v w}-q^{u v w}}{p-q} \text { if } u, v, w \geq 1,
$$

where $0<q<p \leq 1$. The $(p, q)$-factorial is defined by

$$
\begin{gathered}
{[0]_{p, q} !:=1 \text { and }[u v w] !_{p, q}=[1]_{p, q}[2]_{p, q} \Delta \Delta \Delta[u v w]_{p, q} \text { if } u, v, w \geq 1 . \text { and }} \\
u, v, w, m, n, k \in \mathbf{N} .
\end{gathered}
$$

Also the $(p, q)$-binomial coefficient is defined by

$$
\left(\begin{array}{l}
u \\
m
\end{array}\right)\left(\begin{array}{l}
v \\
n
\end{array}\right)\left(\begin{array}{l}
w \\
k
\end{array}\right)_{p, q}=\frac{[u] !_{p, q}}{[m] !_{p, q}[u-m] !_{p, q}} \frac{[v] !_{p, q}}{[n] !_{p, q}[v-n] !_{p, q}} \frac{[w] !_{p, q}}{[k] !_{p, q}[w-k] !_{p, q}}
$$

for all $u, v, w, m, n, k \in \mathbf{N}$ with $(u, v, w) \geq(m, n, k)$.

The formula for $(p, q)$-binomial expansion is as follows:

$$
\begin{aligned}
& (a x+b y)_{p, q}^{u v w} \\
=\sum_{m=0}^{u} \sum_{n=0}^{v} \sum_{k=0}^{w} p^{\frac{(u-m)(u-m-1)+(v-n)(v-n-1)+(w-k)(w-k-1)}{2}} q^{\frac{m(m-1)+n(n-1)+k(k-1)}{2}} & \left(\begin{array}{c}
u \\
m
\end{array}\right)\left(\begin{array}{c}
v \\
n
\end{array}\right)\left(\begin{array}{c}
w \\
k
\end{array}\right)_{p, q} a^{(u-m)+(v-n)+(w-k)} b^{m+n+k} x^{(u-m)+(v-n)+(w-k)} y^{m+n+k} \\
& (x+y)_{p, q}^{u v w}=(x+y)(p x+q y)\left(p^{2} x+q^{2} y\right) \\
\cdots & \left(p^{(u-1)+(v-1)+(w-1)} x+q^{(u-1)+(v-1)+(w-1)} y\right) \\
& (1-x)_{p, q}^{u v w}=(1-x)(p-q x)\left(p^{2}-q^{2} x\right) \\
\cdots & \left(p^{(u-1)+(v-1)+(w-1)}-q^{(u-1)+(v-1)+(w-1)} x\right), \text { and } \\
& (x)_{p, q}^{m n k}=x(p x)\left(p^{2} x\right) \cdots\left(p^{(u-1)+(v-1)+(w-1)} x\right)=p^{\frac{m(m-1)+n(n-1)+k(k-1)}{2}} .
\end{aligned}
$$

The Bernstein operator of order $(r, s, t)$ is given by 


$$
B_{r s t}(f, x)=\sum_{m=0}^{r} \sum_{n=0}^{s} \sum_{k=0}^{t} f\left(\frac{m n k}{r s t}\right)(\stackrel{r}{m})\left(\begin{array}{l}
s \\
n
\end{array}\right)\left(\begin{array}{l}
t \\
k
\end{array}\right) x^{m+n+k}(1-x)^{(m-r)+(n-s)+(k-t)}
$$

where $f$ is a continuous (real or complex valued) function defined on $[0,1]$.

The $(p, q)-$ Bernstein operators are defined as follows:

$$
B_{r s t, p, q}(f, x)=
$$

$$
\begin{aligned}
& 1 \frac{r(r-1)+s(s-1)+t(t-1)}{2} \\
& \sum_{m=0}^{r} \sum_{n=0}^{s} \sum_{k=0}^{t}\left(\begin{array}{l}
m \\
m
\end{array}\right)\left(\begin{array}{l}
s \\
n
\end{array}\right)\left(\begin{array}{l}
t \\
k
\end{array}\right) p^{\frac{m(m-1)+n(n-1)+k(k-1)}{2}} x^{m+n+k} \\
& \prod_{u_{1}=0}^{(r-m-1)}\left(p^{u_{1}}-q^{u_{1}} x\right) \prod_{u_{2}=0}^{(s-n-1)}\left(p^{u_{2}}-q^{u_{2}} x\right) \prod_{u_{3}=0}^{(t-k-1)}\left(p^{u_{3}}-q^{u_{3}} x\right) \\
& \mathrm{f}\left(\frac{[m]_{p, q}[n]_{p, q}[k]_{p, q}}{p^{(m-r)+(n-s)+(k-t)[r]_{p, q}[s]_{p, q}[t]_{p, q}+\mu}}\right), x \in[0,1] \cdots *
\end{aligned}
$$

Also, we have $(1-x)_{p, q}^{r s t}=$

$$
\begin{gathered}
p^{\frac{\sum_{m=0}^{r}(r-m)(r-m-1)+(s-n)(s-n-1)+(t-k)(t-k-1)}{6}} q^{\frac{m(m-1)+n(n-1)+k(k-1)}{6}} \\
\left(\begin{array}{c}
r \\
m
\end{array}\right)\left(\begin{array}{l}
s \\
n
\end{array}\right)\left(\begin{array}{l}
t \\
k
\end{array}\right) x^{m+n+k} .
\end{gathered}
$$

$(p, q)$-Bernstein-Stancu operators are defined as follows:

$$
\begin{aligned}
& S_{r s t, p, q}(f, x)= \\
& 1 \frac{r(r-1)+s(s-1)+t(t-1)}{6} \\
& p^{\frac{r\left(x^{\prime}\right.}{6}} \\
& \sum_{m=0}^{r} \sum_{n=0}^{s} \sum_{k=0}^{t}(\stackrel{r}{m})\left(\begin{array}{l}
s \\
n
\end{array}\right)\left(\begin{array}{l}
t \\
k
\end{array}\right) p^{\frac{m(m-1)+n(n-1)+k(k-1)}{2}} x^{m+n+k} \\
& \prod_{u_{1}=0}^{(r-m-1)}\left(p^{u_{1}}-q^{u_{1}} x\right) \prod_{u_{2}=0}^{(s-n-1)}\left(p^{u_{2}}-q^{u_{2}} x\right) \prod_{u_{3}=0}^{(t-k-1)}\left(p^{u_{3}}-q^{u_{3}} x\right) \\
& \mathrm{f}\left(\frac{p^{(r-m)+(s-n)+(t-k)}[m]_{p, q}[n]_{p, q}[k]_{p, q}+\eta}{[r]_{p, q}[s]_{p, q}[t]_{p, q}+\mu}\right), x \in[0,1] \cdots * *
\end{aligned}
$$

Note that for $\eta=\mu=0,(p, q)$-Bernstein-Stancu operators given by $(* *)$ reduces into $(p, q)-$ Bernstein-Stancu operators. Also for $p=$ $1,(p, q)$-Bernstein-Stancu operators given by $(*)$ turn out to be $q$-Bernstein- 
Stancu operators.

Throughout the paper, $\mathbf{R}$ denotes the real with metric $(X, d)$. Consider a triple sequence of Bernstein stancu polynomials $\left(B_{m n k}(f, x)\right)$ such that $\left(B_{m n k}(f, x)\right) \in \mathbf{R}, m, n, k \in \mathbf{N}^{3}$.

Let $f$ be a continuous function defined on the closed interval $[0,1]$. A triple sequence of Bernstein-Stancu polynomials $\left(S_{r s t, p, q}(f, x)\right)$ is said to be statistically convergent to $0 \in \mathbf{R}$, written as $s t-\lim x=0$, provided that the set

$$
K_{\epsilon}:=\left\{(m, n, k) \in \mathbf{N}^{3}:\left|S_{r s t, p, q}(f, x)-(f, x)\right| \geq \epsilon\right\}
$$

has natural density zero for any $\epsilon>0$. In this case, 0 is called the statistical limit of the triple sequence of Bernstein-Stancu polynomials. i.e., $\delta\left(K_{\epsilon}\right)=$ 0 . That is,

$$
\lim _{r, s, t \rightarrow \infty} \frac{1}{p q j}\left|\left\{(m, n, k) \leq(p, q, j):\left|S_{r s t, p, q}(f, x)-(f, x)\right| \geq \epsilon\right\}\right|=0 .
$$

In this case, we write $\delta-\lim S_{r s t, p, q}(f, x)=(f, x)$ or $S_{r s t, p, q}(f, x) \rightarrow^{S_{S}}$ $(f, x)$.

The theory of statistical convergence has been discussed in trigonometric series, summability theory, measure theory, turnpike theory, approximation theory, fuzzy set theory and so on.

A triple sequence (real or complex) can be defined as a function $x$ : $\mathbf{N} \times \mathbf{N} \times \mathbf{N} \rightarrow \mathbf{R}(\mathbf{C})$, where $\mathbf{N}, \mathbf{R}$ and $\mathbf{C}$ denote the set of natural numbers, real numbers and complex numbers respectively. The different types of notions of triple sequence was introduced and investigated at the initial by Sahiner et al. [15,16], Esi et al. [3-8], Datta et al. [9],Subramanian et al. [17], Debnath et al. [10] and many others.

A triple sequence $x=\left(x_{m n k}\right)$ is said to be triple analytic if

$$
\sup _{m, n, k}\left|x_{m n k}\right|^{\frac{1}{m+n+k}}<\infty .
$$

The space of all triple analytic sequences are usually denoted by $\Lambda^{3}$.

The set of fuzzy real numbers is denoted by $(f, X)(\mathbf{R})$, and $d$ denotes the supremum metric on $(f, X)\left(\mathbf{R}^{3}\right)$. Now let $r$ be nonnegative 
real number. A triple sequence space of Bernstein-Stancu polynomials of $\left(S_{r s t, p, q}(f, X)\right)$ of fuzzy numbers is $r$ - convergent to a fuzzy number $(f, X)$ and we write

$$
S_{r s t, p, q}(f, X) \rightarrow^{r}(f, X) \text { as } m, n, k \rightarrow \infty,
$$

provided that for every $\epsilon>0$ there is an integer $m_{\epsilon}, n_{\epsilon}, k_{\epsilon}$ so that

$$
d\left(S_{r s t, p, q}(f, X),(f, X)\right)<r+\epsilon \text { whenever } m \geq m_{\epsilon}, n \geq n_{\epsilon}, k \geq k_{\epsilon} .
$$

The set $L I M^{r} S_{r s t, p, q}(f, X):=\left\{(f, X) \in(f, X)\left(\mathbf{R}^{3}\right)\right.$ $: S_{r s t, p, q}(f, X) \rightarrow^{r}(f, X)$, as $m, n, k \rightarrow \infty$ is called the $r$ - limit set of the triple sequence space of Bernstein-Stancu polynomials of $\left(S_{r s t, p, q}(f, X)\right)$.

A triple sequence space of Bernstein-Stancu polynomials of fuzzy numbers which is divergent can be convergent with a certain roughness degree. For instance, let us define

$$
S_{r s t, p, q}(f, X)=\left\{\begin{array}{lc}
\eta(X), & \text { if }(m, n, k) \text { are odd integers }, \\
\mu(X), & \text { otherwise }
\end{array}\right\}
$$

where

$$
\eta(X)=\left\{\begin{array}{cc}
X, & \text { if } X \in[0,1], \\
-X+2, & \text { if } X \in[1,2], \\
0, & \text { otherwise }
\end{array}\right\}
$$

and

$$
\mu(X)=\left\{\begin{array}{cc}
X-3, & \text { if } X \in[3,4], \\
-X+5, & \text { if } X \in[4,5], \\
0, & \text { otherwise }
\end{array}\right\}
$$

Then we have where

$$
L I M^{r} S_{r s t, p, q}(f, X)=\left\{\begin{array}{cc}
\phi, & \text { if } r<\frac{3}{2}, \\
{\left[\mu-r_{1}, \eta+r_{1}\right],} & \text { otherwise }
\end{array}\right\},
$$


where $r_{1}$ is nonnegative real number with

$$
\begin{gathered}
{\left[\mu-r_{1}, \eta+r_{1}\right]:=\left\{S_{r s t, p, q}(f, X) \in(f, X)\left(\mathbf{R}^{3}\right)\right.} \\
: \mu-r_{1} \leq S_{r s t, p, q}(f, X) \leq \eta+r_{1} .
\end{gathered}
$$

The ideal of rough convergence of a triple sequence space of BernsteinStancu polynomials can be interpreted as follows:

Let $\left(S_{r s t, p, q}(f, Y)\right)$ be a convergent triple sequence space of BernsteinStancu polynomials of fuzzy numbers. Assume that $\left(S_{r s t, p, q}(f, Y)\right)$ cannot be determined exactly for every $(m, n, k) \in \mathbf{N}^{3}$. That is, $\left(S_{r s t, p, q}(f, Y)\right)$ cannot be calculated so we can use approximate value of $\left(S_{r s t, p, q}(f, Y)\right)$ for simplicity of calculation. We only know that $\left(S_{r s t, p, q}(f, Y)\right) \in\left[\mu_{m n k}, \lambda_{m n k}\right]$, where $d\left(\mu_{m n k}, \lambda_{m n k}\right) \leq r$ for every $(m, n, k) \in \mathbf{N}^{3}$. The triple sequence space of Bernstein-Stancu polynomials of $\left(S_{r s t, p, q}(f, X)\right)$ satisfying

$\left(S_{r s t, p, q}(f, X)\right) \in\left[\mu_{m n k}, \lambda_{m n k}\right]$, for all $m, n, k$. Then the triple sequence space of Bernstein-Stancu polynomials of $\left(S_{r s t, p, q}(f, X)\right)$ may not be convergent, but the inequality

$$
\begin{gathered}
d\left(S_{r s t, p, q}(f, X),(f, X)\right) \leq d\left(S_{r s t, p, q}(f, X), S_{r s t, p, q}(f, Y)\right)+ \\
d\left(S_{r s t, p, q}(f, Y),(f, Y)\right) \leq r+d\left(S_{r s t, p, q}(f, Y),(f, Y)\right)
\end{gathered}
$$

implies that the triple sequence space of Bernstein-Stancu polynomials of $\left(S_{r s t, p, q}(f, X)\right)$ is $r$ - convergent.

In this paper, we first define the concept of rough convergence of a triple sequence space of Bernstein-Stancu polynomials of fuzzy numbers. Also obtain the relation between the set of rough limit and the extreme limit points of a triple sequence space of Bernstein-Stancu polynomials of fuzzy numbers. We show that the rough limit set of a triple sequence space of Bernstein-Stancu polynomials is closed, bounded and convex.

\section{Definitions and Preliminaries}

A fuzzy number $X$ is a fuzzy subset of the real $\mathbf{R}^{3}$, which is normal fuzzy convex, upper semi-continuous, and the $X^{0}$ is bounded where $X^{0}$; = $c l\left\{x \in \mathbf{R}^{3}: X(x)>0\right\}$ and $c l$ is the closure operator. These properties imply that for each $\alpha \in(0$,$] , the \alpha$ - level set $X^{\alpha}$ defined by

$$
X^{\alpha}=\left\{x \in \mathbf{R}^{3}: X(x) \geq \alpha\right\}=\left[\underline{X}^{\alpha}, \bar{X}^{\alpha}\right]
$$


is a non empty compact convex subset of $\mathbf{R}^{3}$.

The supremum metric $d$ on the set $L\left(\mathbf{R}^{3}\right)$ is defined by

$$
d(X, Y)=\sup _{\alpha \in[0,1]} \max \left(\left|\underline{X}^{\alpha}-\underline{Y}^{\alpha}\right|,\left|\bar{X}^{\alpha}-\bar{Y}^{\alpha}\right|\right) .
$$

Now, given $X, Y \in L\left(\mathbf{R}^{3}\right)$, we define $X \leq Y$ if $\underline{X}^{\alpha} \leq \underline{Y}^{\alpha}$ and $\bar{X}^{\alpha} \leq \bar{Y}^{\alpha}$ for each $\alpha \in[0$,$] .$

We write $X \leq Y$ if $X \leq Y$ and there exists an $\alpha_{0} \in[0,1]$ such that $\underline{X}^{\alpha_{0}} \leq \underline{Y}^{\alpha_{0}}$ or $\bar{X}^{\alpha_{0}} \leq \bar{Y}^{\alpha_{0}}$.

A subset $E$ of $L\left(\mathbf{R}^{3}\right)$ is said to be bounded above if there exists a fuzzy number $\mu$, called an upper bound of $E$, such that $X \leq \mu$ for every $X \in E$. $\mu$ is called the least upper bound of $E$ if $\mu$ is an upper bound and $\mu \leq \mu^{\prime}$ for all upper bounds $\mu^{\prime}$.

A lower bound and the greatest lower bound are defined similarly. $E$ is said to be bounded if it is both bounded above and below.

The notions of least upper bound and the greatest lower bound have been defined only for bounded sets of fuzzy numbers. If the set $E \subset L\left(\mathbf{R}^{3}\right)$ is bounded then its supremum and infimum exist.

The limit infimum and limit supremum of a triple sequence spaces $\left(X_{m n k}\right)$ is defined by

$$
\begin{aligned}
\lim _{m n k \rightarrow \infty} \inf X_{m n k} & :=\inf A_{X} . \\
\lim _{m n k \rightarrow \infty} \sup X_{m n k} & :=\inf B_{X} .
\end{aligned}
$$

where

$$
\begin{aligned}
& A_{X}:=\left\{\mu \in L\left(\mathbf{R}^{3}\right): \text { The set }\left\{(m, n, k) \in \mathbf{N}^{3}: X_{m n k}<\mu\right\} \text { is infinite }\right\} \\
& B_{X}:=\left\{\mu \in L\left(\mathbf{R}^{3}\right): \text { The set }\left\{(m, n, k) \in \mathbf{N}^{3}: X_{m n k}>\mu\right\} \text { is infinite }\right\} .
\end{aligned}
$$

Now, given two fuzzy numbers $X, Y \in L\left(\mathbf{R}^{3}\right)$, we define their sum as $Z=X+Y$, where $\underline{Z}^{\alpha}:=\underline{X}^{\alpha}+\underline{Y}^{\alpha}$ and $\bar{Z}^{\alpha}:=\bar{X}^{\alpha}+\bar{Y}^{\alpha}$ for all $\alpha \in[0,1]$.

To any real number $a \in \mathbf{R}^{3}$, we can assign a fuzzy number $a_{1} \in L\left(\mathbf{R}^{3}\right)$, which is defied by 
On rough convergence of triple sequence spaces of Bernstein-Stancu..721

$$
a_{1}(x)=\left\{\begin{array}{cc}
1, & \text { if } x=a, \\
0, & \text { otherwise }
\end{array}\right\}
$$

An order interval in $L\left(\mathbf{R}^{3}\right)$ is defined by $[X, Y]:=\left\{Z \in L\left(\mathbf{R}^{3}\right): X \leq Z \leq Y\right\}$, where $X, Y \in L\left(\mathbf{R}^{3}\right)$.

A set $E$ of fuzzy numbers is called convex if $\lambda \mu_{1}+(1-\lambda) \mu_{2} \in E$ for all $\lambda \in[0,1]$ and $\mu_{1}, \mu_{2} \in E$.

\section{Main Results}

\subsection{Theorem}

Let $f$ be a continuous function defined on the closed interval $[0,1]$. A triple sequence of Bernstein-Stancu polynomials of $\left(S_{r s t, p, q}(f, X)\right)$ of real numbers. If $(f, X) \in L I M^{r} S_{r s t, p, q}(f, X)$, then

$\operatorname{diam}\left(\lim \sup S_{r s t, p, q}(f, X),(f, X)\right) \leq r$ and

$$
\operatorname{diam}\left(\lim \inf S_{r s t, p, q}(f, X),(f, X)\right) \leq r
$$

Proof: We assume that $\operatorname{diam}\left(\lim \sup S_{r s t, p, q}(f, X),(f, X)\right)>r$.

Define $\tilde{\epsilon}:=\frac{\left(\lim \sup S_{r s t, p, q}(f, X),(f, X)\right)-r}{2}$. By definition of limit supremem, we have that given $m_{\tilde{\epsilon}}^{\prime}, n_{\tilde{\epsilon}}^{\prime}, k_{\tilde{\epsilon}}^{\prime} \in \mathbf{N}^{3}$ there exists an $(m, n, k) \in \mathbf{N}^{3}$ with $m \geq m_{\tilde{\epsilon}}^{\prime}, n \geq n_{\tilde{\epsilon}}^{\prime}, k \geq k_{\tilde{\epsilon}}^{\prime}$ such that $\operatorname{diam}\left(\lim \sup S_{r s t, p, q}(f, X),(f, X)\right) \leq \tilde{\epsilon}$. Also, since $S_{r s t, p, q}(f, X) \rightarrow^{r}(f, X)$ as $m, n, k \rightarrow \infty$, there is an integer $m_{\tilde{\epsilon}}^{\prime \prime}, n_{\tilde{\epsilon}}^{\prime \prime}, k_{\tilde{\epsilon}}^{\prime \prime}$ so that

$$
d\left(S_{r s t, p, q}(f, X),(f, X)\right)<r+\tilde{\epsilon}
$$

whenever $m \geq m_{\tilde{\epsilon}}^{\prime \prime}, n \geq n_{\tilde{\epsilon}}^{\prime \prime}, k \geq k_{\tilde{\epsilon}}^{\prime \prime}$. Let

$$
\left(m_{\tilde{\epsilon}}, n_{\tilde{\epsilon}}, k_{\tilde{\epsilon}}\right):=\max \left\{\left(m_{\tilde{\epsilon}}^{\prime}, n_{\tilde{\epsilon}}^{\prime}, k_{\tilde{\epsilon}}^{\prime}\right),\left(m_{\tilde{\epsilon}}^{\prime \prime}, n_{\tilde{\epsilon}}^{\prime \prime}, k_{\tilde{\epsilon}}^{\prime \prime}\right)\right\}
$$

There exists $(m, n, k) \in \mathbf{N}^{3}$ such that $m \geq m_{\tilde{\epsilon}}, n \geq n_{\tilde{\epsilon}}, k \geq k_{\tilde{\epsilon}}$ and

$\operatorname{diam}\left(\lim \sup S_{r s t, p, q}(f, X),(f, X)\right) \leq(f, X) \operatorname{diam}\left(\lim \sup S_{r s t, p, q}(f, X)\right.$, $\mathrm{S}_{r s t, p, q}(f, X)+\operatorname{diam}\left(S_{r s t, p, q},(f, X)\right)$ 


$$
\begin{aligned}
& <\tilde{\epsilon}+r+\tilde{\epsilon} \\
& <r+2 \tilde{\epsilon} \\
& =r+\operatorname{diam}\left(\text { lim sup } S_{r s t, p, q}(f, X),(f, X)\right)-r \\
& =\operatorname{diam}\left(\lim \sup S_{r s t, p, q}(f, X),(f, X)\right) .
\end{aligned}
$$

The contradiction proves the theorem. Similarly,

$\operatorname{diam}\left(\lim \inf S_{r s t, p, q}(f, X),(f, X)\right) \leq r$ can be proved using definition of limit infimum.

\subsection{Theorem}

Let $f$ be a continuous function defined on the closed interval $[0,1]$. A triple sequence of Bernstein-Stancu polynomials of $\left(S_{r s t, p, q}(f, X)\right)$ of real numbers. If $L I M^{r} S_{r s t, p, q}(f, X) \neq \phi$, then we have

$L I M^{r} S_{r s t, p, q}(f, X) \subseteq\left[\left(\limsup S_{r s t, p, q}(f, X)\right)-r_{1},\left(\liminf S_{r s t, p, q}(f, X)\right)+r_{1}\right]$.

Proof: To prove that

$(f, X) \in\left[\left(\limsup S_{r s t, p, q}(f, X)\right)-r_{1},\left(\liminf S_{r s t, p, q}(f, X)\right)+r_{1}\right]$ for an arbitrary $(f, X) \in L I M^{r} S_{r s t, p, q}(f, X)$, i.e.,

$$
\left(\limsup S_{r s t, p, q}(f, X)\right)-r_{1} \leq(f, X) \leq\left(\liminf S_{r s t, p, q}(f, X)\right)+r_{1} .
$$

Let us assume that $\left(\right.$ limsup $\left.S_{r s t, p, q}(f, X)\right)-r_{1} \leq(f, X)$ does not hold. Thus, there exists an $\alpha_{0} \in[0,1]$ such that

$$
\begin{gathered}
\left.\left({\underline{\limsup S_{r s t, p, q}(f, X)}}^{\alpha_{0}}\right)-r_{1}>{\frac{(f, X)}{\overline{l i m s u p}_{m n k}(f, X)}}^{\alpha_{0}}\right)-r_{1}>\leq \overline{(f, X)}^{\alpha_{0}} \\
\text { or }
\end{gathered}
$$

holds i.e.,

$$
\begin{aligned}
& \left.\left(\underline{\limsup }_{\text {rst }, p, q}(f, X)\right)^{\alpha_{0}}\right)-\underline{(f, X)}^{\alpha_{0}}>r_{1} \text { or } \\
& \left({\overline{\limsup S_{r s t, p, q}(f, X)}}^{\alpha_{0}}\right)-\leq \overline{(f, X)}^{\alpha_{0}}>r_{1} \text {. }
\end{aligned}
$$

On the other hand, by theorem (3.1) we have 


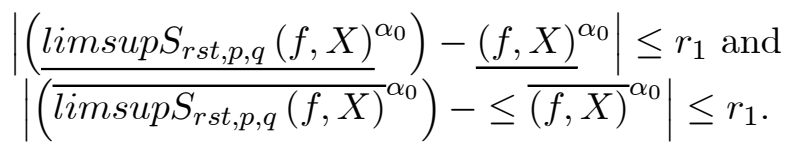

We obtain a contradiction. Hence we get

$\left(\right.$ limsup $\left.S_{r s t, p, q}(f, X)\right)-r_{1} \leq(f, X)$. By using the similar arguments and get it for second part.

\subsection{Note}

The converse inclusion in this theorem holds for $f$ be a continuous function defined on the closed interval $[0,1]$. A triple sequence of Bernstein-Stancu polynomials of $\left(S_{r s t, p, q}(f, X)\right)$ of real numbers, but it may not hold for triple sequences of Bernstein-Stancu polynomials of fuzzy numbers as in the following example:

Example: Define

$$
S_{r s t, p, q}(f, X)=\left\{\begin{array}{cc}
\frac{-1}{2(m n k)} X+1, & \text { if } X \in[0,1], \\
0, & \text { otherwise }
\end{array}\right\}
$$

and

$$
(f, X)=\left\{\begin{array}{cc}
1, & \text { if } X \in[0,1] \\
0, & \text { otherwise }
\end{array}\right\}
$$

Then we have $\left|\overline{(f, X)}^{1}-{\overline{S_{r s t, p, q}(f, X)}}^{1}\right|=|1-0|=1$, i.e., $d\left(S_{r s t, p, q}(f, X),(f, X)\right) \geq 1$ for all $(m, n, k) \in \mathbf{N}^{3}$. Although the triple sequence spaces of Bernstein-Stancu poynomials of $\left(S_{r s t, p, q}(f, X)\right)$ is not convergent to $(f, X)$, limsup $S_{r s t, p, q}(f, X)$ and liminf $S_{r s t, p, q}(f, X)$ of this triple sequence space of Bernstein-Stancu polynomials are equal to $(f, X)$. Hence we get

$$
\begin{gathered}
L \in\left[\limsup S_{r s t, p, q}(f, X)-\left(\frac{1}{2}\right)_{1}, \liminf S_{r s t, p, q}(f, X)+\left(\frac{1}{2}\right)_{1}\right], \text { but } \\
(f, X) \notin L I M^{\frac{1}{2}} S_{r s t, p, q}(f, X) .
\end{gathered}
$$




\subsection{Theorem}

Let $f$ be a continuous function defined on the closed interval $[0,1]$. A triple sequence of Bernstein-Stancu polynomials of $\left(S_{r s t, p, q}(f, X)\right)$ of real numbers converges to the fuzzy number $(f, X)$, then

$$
L I M^{r} S_{r s t, p, q}(f, X)=\bar{S}_{r}((f, X)):=\left\{\mu \in(f, X)\left(\mathbf{R}^{3}\right): d(\mu,(f, X)) \leq r\right\}
$$

Proof: Let $\epsilon>0$. Since the triple sequence space of Bernstein-Stancu polynomials of $\left(S_{r s t, p, q}(f, X)\right)$ is convergent to $(f, X)$, there is an integer $m_{\epsilon}, n_{\epsilon}, k_{\epsilon}$ so that

$$
d\left(S_{r s t, p, q}(f, X),(f, X)\right)<\epsilon \text { whenever } m \geq m_{\epsilon}, n \geq n_{\epsilon}, k \geq k_{\epsilon} .
$$

Let $Y \in \bar{S}_{r}((f, X))$, we have

$$
\begin{gathered}
d\left(S_{r s t, p, q}(f, X), Y\right) \leq d\left(S_{r s t, p, q}(f, X),(f, X)\right)+d((f, X), Y)<\epsilon+r \text { for } \\
\text { every } m \geq m_{\epsilon}, n \geq n_{\epsilon}, k \geq k_{\epsilon} .
\end{gathered}
$$

Hence we have $Y \in L I M^{r} S_{r s t, p, q}(f, X)$.

Now let $Y \in L I M^{r} S_{r s t, p, q}(f, X)$. Hence there is an integer $m_{\epsilon}^{\prime}, n_{\epsilon}^{\prime}, k_{\epsilon}^{\prime}$ so that

$$
d\left(S_{r s t, p, q}(f, X), Y\right)<r+\epsilon
$$

whenever $m \geq m_{\epsilon}^{\prime}, n \geq n_{\epsilon}^{\prime}, k \geq k_{\epsilon}^{\prime}$. Let

$$
\left(m_{\epsilon}^{\prime \prime}, n_{\epsilon}^{\prime \prime}, k_{\epsilon}^{\prime \prime}\right):=\max \left\{\left(m_{\epsilon}, n_{\epsilon}, k_{\epsilon}\right),\left(m_{\epsilon}^{\prime}, n_{\epsilon}^{\prime}, k_{\epsilon}^{\prime}\right)\right\}
$$

for all $m \geq m_{\epsilon}^{\prime \prime}, n \geq n_{\epsilon}^{\prime \prime}, k \geq k_{\epsilon}^{\prime \prime}$, we obtain

$$
\begin{gathered}
d(Y, f(X)) \leq d\left(Y, S_{r s t, p, q}(f, X)\right)+d\left(S_{r s t, p, q}(f, X),\right. \\
(f, X)<r+\epsilon+\epsilon<r+2 \epsilon .
\end{gathered}
$$

Since $\epsilon$ is arbitrary, we have $d(Y,(f, X)) \leq r$. Hence we get $Y \in$ $\bar{S}_{r}((f, X))$. Thus, if the triple sequence space of Bernstein-Stancu polynomials of $\left(S_{r s t, p, q}(f, X)\right) \rightarrow^{r}(f, X)$, then $L I M^{r} S_{r s t, p, q}(f, X)=\bar{S}_{r}((f, X))$. 


\subsection{Theorem}

Let $f$ be a continuous function defined on the closed interval $[0,1]$. A triple sequence of real numbers of Bernstein -Stancu polynomials of $\left(S_{r s t, p, q}(f, X)\right)$ and $\left(S_{r s t, p, q}(f, Y)\right) \in(f, X)\left(\mathbf{R}^{3}\right)$. If $S_{r s t, p, q}(f, X) \rightarrow^{r}(f, X)$ then $S_{r s t, p, q}(f, Y) \rightarrow^{r}(f, Y)$ and $d\left(S_{r s t, p, q}(f, X), S_{r s t, p, q}(f, Y)\right) \leq r$ for every $(m, n, k) \in \mathbf{N}^{3}$.

Proof: Assume that $S_{r s t, p, q}(f, Y) \rightarrow^{r}(f, Y)$, as $m, n, k \rightarrow \infty$ and

$$
d\left(S_{r s t, p, q}(f, X), S_{r s t, p, q}(f, Y)\right) \leq r \text { for every }(m, n, k) \in \mathbf{N}^{3} \text {. We have }
$$

$S_{m n k}(f, Y) \rightarrow^{r}(f, Y)$, as $m, n, k \rightarrow \infty$ means that for every $\epsilon>0$ there exists an $m_{\epsilon}, n_{\epsilon}, k_{\epsilon}$ such that

$$
d\left(S_{r s t, p, q}(f, Y),(f, Y)\right)<\epsilon \text { for all } m \geq m_{\epsilon}, n \geq n_{\epsilon}, k \geq k_{\epsilon}
$$

If the in equality $d\left(S_{r s t, p, q}(f, X), S_{r s t, p, q}(f, Y)\right) \leq r$ yields then

$$
\begin{gathered}
d\left(S_{r s t, p, q}(f, X),(f, X)\right) \leq \\
d\left(S_{r s t, p, q}(f, X), S_{r s t, p, q}(f, Y)\right)+d\left(S_{r s t, p, q}(f, Y),(f, Y)\right)<r+\epsilon \text { for all } \\
m \geq m_{\epsilon}, n \geq n_{\epsilon}, k \geq k_{\epsilon} .
\end{gathered}
$$

Hence the triple sequence space of Bernstein-Stancu polynomials of $\left(S_{r s t, p, q}(f, X)\right)$ is $r$ - convergent to the fuzzy number $(f, X)$.

\subsection{Theorem}

Let $f$ be a continuous function defined on the closed interval $[0,1]$. A triple sequence of Bernstein-Stancu polynomials of $\left(S_{r s t, p, q}(f, X)\right)$ of real numbers and the diameter of an $r$ - limit set is not greater than $3 r$.

Proof: We have to prove that

$$
\sup \left\{d(W, Z): W, Y, Z \in L I M^{r} S_{r s t, p, q}(f, X)\right\} \leq 3 r .
$$

Assume on the contrary that

$$
\sup \left\{d(W, Z): W, Y, Z \in L I M^{r} S_{r s t, p, q}(f, X)\right\}>3 r .
$$


By this assumption, there exists,

$W, Y, Z \in L I M^{r} S_{r s t, p, q}(f, X)$ satisfying $\lambda:=d(W, Z)>3 r$. For an arbitrary $\epsilon \in\left(0, \frac{\lambda}{3}-r\right)$, we have

$\exists\left(m_{\epsilon}^{\prime}, n_{\epsilon}^{\prime}, k_{\epsilon}^{\prime}\right) \in \mathbf{N}^{3}: \forall(m, n, k) \geq\left(m_{\epsilon}^{\prime}, n_{\epsilon}^{\prime}, k_{\epsilon}^{\prime},\right) \Longrightarrow d\left(S_{r s t, p, q}(f, X), W\right)$ $\leq r+\epsilon$,

$\exists\left(m_{\epsilon}^{\prime \prime}, n_{\epsilon}^{\prime \prime}, k_{\epsilon}^{\prime \prime}\right) \in \mathbf{N}^{3}: \forall(m, n, k) \geq\left(m_{\epsilon}^{\prime \prime}, n_{\epsilon}^{\prime \prime}, k_{\epsilon}^{\prime \prime},\right)\left(S_{r s t, p, q}(f, X), Y\right)$ $\leq r+\epsilon$,

$\exists\left(m_{\epsilon}^{\prime \prime \prime}, n_{\epsilon}^{\prime \prime \prime}, k_{\epsilon}^{\prime \prime \prime}\right) \in \mathbf{N}^{3}: \forall(m, n, k) \geq\left(m_{\epsilon}^{\prime \prime \prime}, n_{\epsilon}^{\prime \prime \prime}, k_{\epsilon}^{\prime \prime \prime},\right) \Longrightarrow d\left(S_{r s t, p, q}(f, X), Z\right)$ $\leq r+\epsilon$.

Define $\left(m_{\epsilon}, n_{\epsilon}, k_{\epsilon}\right):=\max \left\{\left(m_{\epsilon}^{\prime}, n_{\epsilon}^{\prime}, k_{\epsilon}^{\prime}\right),\left(m_{\epsilon}^{\prime \prime}, n_{\epsilon}^{\prime \prime}, k_{\epsilon}^{\prime \prime}\right),\left(m_{\epsilon}^{\prime \prime \prime}, n_{\epsilon}^{\prime \prime \prime}, k_{\epsilon}^{\prime \prime \prime}\right)\right\}$.

Thus we get

$$
\begin{aligned}
d(W, Z) \leq & d\left(S_{r s t, p, q}(f, X), W\right)+d\left(S_{r s t, p, q}(f, X), Y\right)+d\left(S_{r s t, p, q}(f, X), Z\right) \\
& <(r+\epsilon)+(r+\epsilon)+(r+\epsilon) \\
& <3(r+\epsilon) \\
& <3 r+3\left(\frac{\lambda}{3}-r\right)<3 r+\lambda-3 r \\
& =\lambda \text { for all } m \geq m_{\epsilon}, n \geq n_{\epsilon}, k \geq k_{\epsilon}
\end{aligned}
$$

which contradicts to the fact that $\lambda=d(W, Z)$.

\subsection{Theorem}

Let $f$ be a continuous function defined on the closed interval $[0,1]$. A triple sequence of Bernstein-Stancu polynomials of $\left(S_{r s t, p, q}(f, X)\right)$ of real numbers is analytic if and only if there exists an $r \geq 0$ such that $L I M^{r} S_{r s t, p, q}(f, X) \neq \phi$.

Proof: Necessity: Let the triple sequence space of Bernstein-Stancu polynomials of $\left(S_{r s t, p, q}(f, X)\right)$ be a analytic sequence and 


$$
s:=\sup \left\{d\left(S_{r s t, p, q}(f, X)^{1 / m+n+k}, 0\right):(m, n, k) \in \mathbf{N}^{3}\right\}<\infty .
$$

Then we have $0 \in L I M^{s} S_{r s t, p, q}(f, X)$, i.e., $L I M^{r} S_{r s t, p, q}(f, X) \neq \phi$, where $r=s$.

Sufficiency: If $L I M^{r} S_{r s t, p, q}(f, X) \neq \phi$ for some $r \geq 0$, then there exists $(f, X) \in L I M^{r} S_{r s t, p, q}(f, X)$. By definition, for every $\epsilon>0$ there is an integer $\left(m_{\epsilon}, n_{\epsilon}, k_{\epsilon}\right)$ so that

$$
d\left(S_{r s t, p, q}(f, X),(f, X)\right)<r+\epsilon \text { whenever } m \geq m_{\epsilon}, n \geq n_{\epsilon}, k \geq k_{\epsilon} .
$$

Define

$$
\begin{gathered}
t=t(\epsilon):= \\
\operatorname{Max}\left\{d((f, X), 0), d\left(S_{111, p, q}(f, X), 0\right), \cdots, d\left(S_{r_{\epsilon} s_{\epsilon} t_{\epsilon}, p, q}(f, X), 0\right), r+\epsilon\right\} .
\end{gathered}
$$

Then we have

$$
S_{r s t, p, q} \in\left\{\mu \in(f, X)\left(\mathbf{R}^{3}\right): d(\mu, 0) \leq t+r+\epsilon\right\} \text { for every }(m, n, k) \in \mathbf{N}^{3} \text {, }
$$

which proves the boundedness of the triple sequence space of BernsteinStancu polynomials of $\left(S_{r s t, p, q}(f, X)\right)$.

\subsection{Theorem}

Let $f$ be a continuous function defined on the closed interval $[0,1]$. A triple sequence of Bernstein-Stancu polynomials of $\left(S_{u_{m} v_{n} w_{k}, p, q}(f, X)\right)$ of real numbers is a sub sequence of a triple sequence space of BernsteinStancu polynomials of $\left(S_{r s t, p, q}(f, X)\right)$, then

$$
L I M^{r} S_{r s t, p, q}(f, X) \subset L I M^{r} S_{u_{m} v_{n} w_{k}, p, q}(f, X) .
$$

\section{Proof: Omitted.}

\subsection{Theorem}

Let $f$ be a continuous function defined on the closed interval $[0,1]$. A triple sequence of Bernstein-Stancu polynomials of $\left(S_{r s t, p, q}(f, X)\right)$ of real numbers, for all $r \geq 0$, the $r$-limit set $L I M^{r} S_{r s t, p, q}(f, X)$ of an arbitrary triple sequence space of Bernstein-Stancu polynomials of $S_{r s t, p, q}(f, X)$ is closed.

Proof: Let $\left(Y_{m n k}\right) \subset L I M^{r} S_{r s t, p, q}(f, Y)$ and $S_{r s t, p, q}(f, Y) \rightarrow(f, Y)$ as $m, n, k \rightarrow \infty$. Let $\epsilon>0$. Since the triple sequence space of BernsteinStancu polynomials of $\left(S_{r s t, p, q}(f, Y)\right) \rightarrow^{r}(f, Y)$, there is an integer $i_{\epsilon} j_{\epsilon} \ell_{\epsilon}$ so that 


$$
d\left(S_{r s t, p, q}(f, Y),(f, Y)\right)<\frac{\epsilon}{2} \text { whenever } m \geq i_{\epsilon}, n \geq j_{\epsilon}, k \geq \ell_{\epsilon} .
$$
so that

Since $S_{i_{\epsilon} j_{\epsilon} \ell_{\epsilon}, p, q}(f, Y) \in L I M^{r} S_{r s t, p, q}(f, X)$, there is an integer $\left(m_{\epsilon} n_{\epsilon} k_{\epsilon}\right)$

$$
\begin{gathered}
d\left(S_{r s t, p, q}(f, X), S_{i_{\epsilon} j_{\epsilon} \ell_{\epsilon}, p, q}(f, Y)\right)<r+\frac{\epsilon}{2} \text { whenever } \\
m \geq m_{\epsilon}, n \geq n_{\epsilon}, k \geq k_{\epsilon} .
\end{gathered}
$$

Therefore, we have

$$
\begin{gathered}
d\left(S_{r s t, p, q}(f, X),(f, X)\right) \leq d\left(S_{r s t, p, q}(f, X), S_{i_{\epsilon} j_{\epsilon} \ell_{\epsilon}, p, q}(f, Y)\right)<r+\frac{\epsilon}{2}+\frac{\epsilon}{2}= \\
r+\epsilon \text { for every } m \geq m_{\epsilon}, n \geq n_{\epsilon}, k \geq k_{\epsilon} .
\end{gathered}
$$

Hence $L \in L I M^{r} S_{r s t, p, q}(f, X)$ implies that the set $L I M^{r} S_{r s t, p, q}(f, X)$ is closed.

\section{Conclusions and Future Work}

We introduced triple sequence space of Bernstein-Stancu polynomials of rough convergence of fuzzy numbers. For the reference sections, consider the following introduction described the main results are motivating the research.

Competing Interests: The authors declare that there is not any conflict of interests regarding the publication of this manuscript.

\section{References}

[1] S. Aytar , Rough statistical convergence, Numer. Funct. Anal. Optimiz, 29 (3-4), (2008), pp. 291-303, (2008).

[2] S. Aytar, The rough limit set and the core of a real sequence, Numer. Funct. Anal. Optimiz, 29 (3-4), pp. 283-290, (2008).

[3] A. Esi , On some triple almost lacunary sequence spaces defined by Orlicz functions, Research and Reviews:Discrete Mathematical Structures, 1 (2), pp. 16-25, (2014).

[4] A. Esi and M. Necdet Catalbas,Almost convergence of triple sequences, Global Journal of Mathematical Analysis, 2 (1), pp. 6-10, (2014). 
[5] A. Esi and E. Savas, On lacunary statistically convergent triple sequences in probabilistic normed space,Appl.Math.and Inf.Sci., 9 (5), pp. 2529-2534, (2015).

[6] A. Esi, S. Araci and M. Acikgoz, Statistical Convergence of Bernstein Operators, Appl.Math.and Inf.Sci., 10 (6), pp. 2083-2086, (2016).

[7] A. Esi, S. Araci and Ayten Esi, $\lambda$-Statistical Convergence of Bernstein polynomial sequences, Advances and Applications in Mathematical Sciences, 16 (3) , (2017), 113-119.

[8] A. Esi, N. Subramanian and Ayten Esi, On triple sequence space of Bernstein operator of rough I- convergence Pre-Cauchy sequences, Proyecciones Journal of Mathematics, 36 (4), pp. 567-587, (2017).

[9] A. J. Datta A. Esi and B.C. Tripathy,Statistically convergent triple sequence spaces defined by Orlicz function, Journal of Mathematical Analysis, 4(2), pp. 16-22, (2013).

[10] S. Debnath, B. Sarma and B.C. Das ,Some generalized triple sequence spaces of real numbers, Journal of Nonlinear Analysis and Optimization, Vol. 6, No. 1, pp. 71-79, (2015).

[11] E. Dündar, C. Cakan, Rough I- convergence, Demonstratio Mathematica, Accepted.

[12] H.X. Phu, Rough convergence in normed linear spaces, Numer. Funct. Anal. Optimiz, 22, pp. 199-222, (2001).

[13] H.X. Phu, Rough continuity of linear operators, Numer. Funct. Anal. Optimiz, 23, pp. 139-146, (2002).

[14] H.X. Phu , Rough convergence in infinite dimensional normed spaces, Numer. Funct. Anal. Optimiz, 24, pp. 285-301, (2003).

[15] A. Sahiner, M. Gurdal and F.K. Duden, Triple sequences and their statistical convergence, Selcuk J. Appl. Math., 8 No. (2), pp. 49-55, (2007).

[16] A. Sahiner, B.C. Tripathy , Some $I$ related properties of triple sequences, Selcuk J. Appl. Math., 9 No. (2), pp. 9-18, (2008).

[17] N. Subramanian and A. Esi, The generalized tripled difference of $\chi^{3}$ sequence spaces, Global Journal of Mathematical Analysis, 3 (2), pp. 54-60, (2015). 


\section{Jeyaram Bharathi}

Department of Mathematics

Hindustan Institute of Technology and Science

Chennai - 603 103,

India

e-mail : jeyarambharathi@yahoo.com

\section{S. Velmurugan}

Department of Mathematics

Hindustan Institute of Technology and Science

Chennai - 603 103,

India

e-mail : ksvelmurugan.09@gmail.com

\section{A. Esi}

Department of Mathematics

Adiyaman University, 02040

Adiyaman,

Turkey

e-mail : aesi23@hotmail.com

and

\section{N. Subramanian}

Department of Mathematics

SASTRA University,

Thanjavur-613 401,

India

e-mail : nsmaths@gmail.com 